\title{
Molecular Characterization of the phaEC $C_{\mathrm{Hm}}$ Genes, Required for Biosynthesis of Poly(3-Hydroxybutyrate) in the Extremely Halophilic Archaeon Haloarcula marismortui ${ }^{\nabla}$
}

\author{
Jing Han, ${ }^{1,2} \dagger$ Qiuhe Lu, ${ }^{1,2} \dagger$ Ligang Zhou, ${ }^{1,2}$ Jian Zhou, ${ }^{1}$ and Hua Xiang ${ }^{1 *}$ \\ State Key Laboratory of Microbial Resources, Institute of Microbiology, Chinese Academy of Sciences, ${ }^{1}$ and \\ Graduate University of Chinese Academy of Sciences, ${ }^{2}$ Beijing, People's Republic of China
}

Received 28 April 2007/Accepted 28 July 2007

\begin{abstract}
Although many haloarchaea produce biodegradable polyhydroxyalkanoates (PHAs), the genes involved in PHA synthesis in the domain of Archaea have not yet been experimentally investigated yet. In this study, we revealed that Haloarcula marismortui was able to accumulate poly(3-hydroxybutyrate) (PHB) up to $21 \%$ of cellular dry weight when cultured in a minimal medium with excessive glucose and identified the $p h a E_{\mathrm{Hm}}$ and pha $C_{\mathrm{Hm}}$ genes, probably encoding two subunits of a class III PHA synthase. These two genes were adjacent and directed by a single promoter located 26 bp upstream of the transcriptional start site and were constitutively expressed under both nutrient-rich and -limited conditions. Interestingly, $\mathrm{PhaC}_{\mathrm{Hm}}$ was revealed to be strongly bound with the PHB granules, but $\mathrm{PhaE}_{\mathrm{Hm}}$ seemed not to be. Introduction of either the phaE $E_{\mathrm{Hm}}$ or phaC gene into Haloarcula hispanica, which harbors highly homologous $p h a E C_{\mathrm{Hh}}$ genes, could enhance the PHB synthesis in the recombinant strains, while coexpression of the both genes always generated the highest PHB yield. Significantly, knockout of the $\mathrm{phaEC}_{\mathrm{Hh}}$ genes in $\mathrm{H}$. hispanica led to a complete loss of the PHA synthase activity. Complementation with phaEC $_{\mathrm{Hm}}$ genes, but not a single one, restored the capability of PHB accumulation as well as the PHA synthase activity in this phaEC-deleted haloarchaeon. These results indicated that the phaEC genes are required for biosynthesis of PHB and might encode an active PHA synthase in the Haloarcula species.
\end{abstract}

Polyhydroxyalkanoates (PHAs) are a class of biodegradable polyesters of $(R)$-hydroxyalkanoates. These water-insoluble biopolymers are accumulated by a wide variety of bacteria and haloarchaea when the carbon source is available in excess but other nutrients are growth limiting (29). PHAs are attracting increasing attention due to their biodegradable, biocompatible, and thermoplastic features; thus, they are potential substitutes for petrochemical-derived plastics and can be used as packaging and biomedical materials, nonwoven fabrics, and flavor delivery agents (26). Nevertheless, the expensive cost for PHA production has limited its large-scale application. In this regard, the haloarchaeal strains employed as PHA producers have many advantages $(17,23)$. First, haloarchaea are a group of extreme archaea growing optimally in hypersaline environments (25). As there are very few microorganisms that are able to survive and grow at high salinities, the risks of microbial contamination can be reduced. Second, some cheaper carbon sources, such as starch, could be used to synthesize PHAs by haloarchaea, and so the cost of PHA production would be lowered. Third, haloarchaea can be easily lysed in distilled water, which could avoid the use of large quantities of organic solvent and save time for PHA preparation. Moreover, although high concentrations of saline minerals in the culture

\footnotetext{
* Corresponding author. Mailing address: State Key Laboratory of Microbial Resources, Institute of Microbiology, Chinese Academy of Sciences, Datun Road, Chaoyang District, Beijing 100101, People's Republic of China. Phone: (86) 10-6480-7472. Fax: (86) 10-6480-7472. E-mail: xiangh@sun.im.ac.cn.

$\dagger$ J.H. and Q.L. contributed equally to this paper.

${ }^{\nabla}$ Published ahead of print on 3 August 2007.
}

medium may cause corrosion of fermentors, a novel corrosionresistant bioreactor has been reported (10). Therefore, PHA production in haloarchaea will have much economic potential after we have a thorough understanding of PHA synthesis in these halophilic archaea.

The presence of PHA granules in haloarchaea was first reported in 1972 (15). Since then, many haloarchaeal genera, including Haloferax, Halobacterium, Haloarcula, and Haloquadratum, have been found to accumulate kinds of PHAs, such as poly(3-hydroxybutyrate) (PHB) or poly(3-hydroxybutyrate-co-hydroxyvalerate) $(6,10,15,17,19)$. Some of these halophilic archaea, including haloarchaeal strain 56 and Haloferax mediterranei ATCC 33500, have been shown to accumulate large amounts of PHA ( $\sim 60 \%$ of cellular dry weight) under optimized culture conditions $(6,10,23)$. However, although the enzymatic properties of PHA synthase from the crude extracts of the halophilic archaeon strain 56 have been investigated (11), the molecular features of the PHA synthase in the domain of Archaea are still far from well characterized. PHA synthases are the central enzymes during the polymerization of 3-hydroxyacyl-coenzyme A (CoA) thioesters into PHAs. The characteristics of PHA synthases are closely related to the monomer composition, molecular weight, and the amount and polymer properties of the generated PHAs (14, 34). At present, PHA synthases from bacteria are grouped into four classes according to their substrate specificity and the subunit compositions (36). The PHA synthase of Allochromatium vinosum (originally named Chromatium vinosum), consisting of two subunits, $\mathrm{PhaE}_{\mathrm{Av}}(40.53 \mathrm{kDa})$ and $\mathrm{PhaC}_{\mathrm{Av}}(39.73$ $\mathrm{kDa}$ (22), is a representative of the class III synthases. Many 
TABLE 1. Strains and plasmids used in this study

\begin{tabular}{|c|c|c|}
\hline Strain or plasmid & Relevant characteristics & Source or reference \\
\hline \multicolumn{3}{|l|}{ Strains } \\
\hline Haloarcula marismortui ATCC 43049 & Wild-type strain & 1 \\
\hline Haloarcula hispanica ATCC 33960 & Wild-type strain & ATCC \\
\hline Haloarcula hispanica PHB-1 & phaEC-deleted mutant of $H$. hispanica & This study \\
\hline Haloferax volcanii DS70 & Wild-type strain cured of endogenous $\mathrm{pHV} 2$ plasmid & 40 \\
\hline Escherichia coli JM109 & recA1 supE44 endA1 hsdR17 gyrA96 relA1 thi & 30 \\
\hline Escherichia coli BL21(DE3) & $\mathrm{F}^{-}$ompT hsd $S_{B}\left(\mathrm{r}_{\mathrm{B}}^{-} \mathrm{m}_{\mathrm{B}}^{-}\right) d c m$ gal (DE3) & Novagen \\
\hline \multicolumn{3}{|l|}{ Plasmids } \\
\hline pWL102 & 10.5-kb shuttle vector, $\mathrm{Amp}^{\mathrm{r}} \mathrm{Mev}^{\mathrm{r}}$ & 18 \\
\hline pWLE & $11.2 \mathrm{~kb}$, phaE and its native promoter & This study \\
\hline pWLfdxC & $12.1 \mathrm{~kb}$, promoter of $f d x$ and phaC & This study \\
\hline pWLEC & $12.6 \mathrm{~kb}$, phaEC and its native promoter & This study \\
\hline pET28a & $5.4 \mathrm{~kb}$, IPTG-inducible expression vector with $\mathrm{His}$ tag & Novagen \\
\hline pET28aE & Expression plasmid for His tag fused to $\mathrm{PhaE}$ & This study \\
\hline pET28aC & Expression plasmid for His tag fused to $\mathrm{PhaC}$ & This study \\
\hline pUBP2 & 12.3-kb shuttle vector, $\mathrm{Amp}^{\mathrm{r}} \mathrm{Mev}^{\mathrm{r}}$ & 2 \\
\hline pUBP & $\begin{array}{l}6.6 \mathrm{~kb} \text {, derivative of pUBP } 2 \text { created by removing the } \\
\text { pHH9-ori region }\end{array}$ & This study \\
\hline pUBPHL & $\begin{array}{l}7.6 \mathrm{~kb} \text {, integration vector for knockout of } p h a E C_{\mathrm{Hh}} \\
\text { of } H \text {. hispanica ATCC } 33960\end{array}$ & This study \\
\hline
\end{tabular}

comprehensive studies of this bacterial PHA synthase have been performed in vivo and in vitro $(13,24,27,38,41)$. In haloarchaea, the genes encoding the homologues of class III PhaC were already annotated in the recently published genome sequences of Haloarcula marismortui ATCC 43049 (1) and Haloquadratum walsbyi DSM 16790 (3). This has provided an opportunity to investigate the PHA synthesis pathways in the haloarchaea.

In this study, we report the molecular characterization of the phaEC $C_{\mathrm{Hm}}$ operon that is required for $\mathrm{PHB}$ synthesis in the extremely halophilic archaeon $H$. marismortui. The expression profile of the phaEC $C_{\mathrm{Hm}}$ genes was analyzed, and the significance of the two genes in PHB synthesis was identified through both heterologous expression and gene knockout/complementation investigations. We suggest that both $\mathrm{PhaC}_{\mathrm{Hm}}$ and $\mathrm{PhaE}_{\mathrm{Hm}}$ are necessary for an active PHA synthase in $H$. marismortui.

\section{MATERIALS AND METHODS}

Strains and growth conditions. The strains used in this study are listed in Table 1. Escherichia coli was grown in Luria-Bertani medium at $37^{\circ} \mathrm{C}(30)$. Unless otherwise noted, H. marismortui, H. hispanica, and Haloferax volcanii were cultivated at $37^{\circ} \mathrm{C}$ in nutrient-rich medium AS-168 (per liter, $5.0 \mathrm{~g}$ Bacto Casamino Acids, $5.0 \mathrm{~g}$ yeast extract, $1.0 \mathrm{~g}$ sodium glutamate, $3.0 \mathrm{~g}$ trisodium citrate, $200 \mathrm{~g}$ $\mathrm{NaCl}, 20 \mathrm{~g} \mathrm{MgSO}_{4} \cdot 7 \mathrm{H}_{2} \mathrm{O}, 2.0 \mathrm{~g} \mathrm{KCl}, 0.36 \mathrm{~g} \mathrm{FeSO}_{4} \cdot 4 \mathrm{H}_{2} \mathrm{O}$, and $0.36 \mathrm{mg} \mathrm{MnCl}_{2}$ $4 \mathrm{H}_{2} \mathrm{O}, \mathrm{pH}$ 7.2). For analysis of PHA accumulation in haloarchaea, nutrientlimited minimal medium (per liter, $200 \mathrm{~g} \mathrm{NaCl}, 20 \mathrm{~g} \mathrm{MgSO}_{4} \cdot 7 \mathrm{H}_{2} \mathrm{O}, 2 \mathrm{~g} \mathrm{KCl}, 1 \mathrm{~g}$ sodium glutamate, $37.5 \mathrm{mg} \mathrm{KH} \mathrm{PO}_{4}, 50 \mathrm{mg} \mathrm{FeSO}{ }_{4} \cdot 7 \mathrm{H}_{2} \mathrm{O}, 0.36 \mathrm{mg} \mathrm{MnCl}_{2}$. $4 \mathrm{H}_{2} \mathrm{O}, 1$ g yeast extract, $\mathrm{pH}$ 7.2) supplemented with $2 \%$ glucose, resulting in the PHA accumulation medium MG, was explored. Cells were first cultivated in AS-168 medium for $72 \mathrm{~h}$ and then transferred into MG medium and continuously cultured for $48 \mathrm{~h}$ on a rotary shaker. Afterwards, $100 \mathrm{ml}$ of these seed cultures was inoculated into 2 liters of MG medium for fermentation in a 5-liter computer-controlled fermentor (NBS Bioflo 110) and cultured for 4 to $\sim 10$ days with respect to $H$. hispanica or $H$. marismortui strains. When needed, $3 \mu \mathrm{g} / \mathrm{ml}$ mevinolin, $100 \mu \mathrm{g} / \mathrm{ml}$ ampicillin, or $50 \mu \mathrm{g} / \mathrm{ml} \mathrm{kanamycin} \mathrm{was} \mathrm{added} \mathrm{into} \mathrm{the}$ medium.

Plasmid construction, transformation, and gene knockout. The plasmids and primers used in this study are listed in Tables 1 and 2, respectively. The expression plasmids for haloarchaea were derived from pWL102, a shuttle vector between $E$. coli and haloarchaea (Table 1). Unless otherwise noted, the inserts were PCR amplified from the genomic DNA of H. marismortui ATCC 43049. Plasmids pWLE and pWLEC were constructed by, respectively, cloning of pha $E_{\mathrm{Hm}}$ (amplified with primers phaEF1 and phaER1) and $p h a E C_{\mathrm{Hm}}$ (amplified with primers phaEF1 and phaCR1) into pWL102, with the native promoter of phaEC $C_{\mathrm{Hm}}$. For overexpression of the pha $C_{\mathrm{Hm}}$ gene, the promoter sequence of the $f d x_{\mathrm{Hm}}$ gene (amplified with primers $\mathrm{fdxF}$ and $\mathrm{fdxR}$ ) and coding sequence of phaC $C_{\mathrm{Hm}}$ (amplified with primers phaCF1 and phaCR1) were inserted into pWL102, resulting in the plasmid pWLfdxC. For expression of $\mathrm{PhaE}_{\mathrm{Hm}}$ and $\mathrm{PhaC}_{\mathrm{Hm}}$ in E. coli $\mathrm{BL} 21(\mathrm{DE} 3)$, pha $E_{\mathrm{Hm}}$ and $p h a C_{\mathrm{Hm}}$ genes were amplified by primer pairs phaEF2/phaER2 and phaCF2/phaCR2 and cloned into the expression vector pET28a, resulting in pET28aE and pET28aC, respectively (Table 1).

For knockout of phaEC $C_{\mathrm{Hh}}$ genes in $H$. hispanica ATCC 33960, a 434-bp DNA fragment located immediately upstream of the $p h a E C_{\mathrm{Hh}}$ operon and a 563-bp fragment at the $3^{\prime}$ region of the $p h a C_{\mathrm{Hh}}$ gene were amplified by primer pairs CrossF1/CrossR1 and CrossF2/CrossR2, respectively (Table 2). These two DNA fragments were linked and inserted into the plasmid pUBP, a derivative of

TABLE 2. Primers used in this study

\begin{tabular}{|c|c|}
\hline Primer & Sequence $\left(5^{\prime} \text { to } 3^{\prime}\right)^{a}$ \\
\hline \multicolumn{2}{|l|}{ fdxF. } \\
\hline fdxR.... & ......GGCGGATCCCGGCATCACTGCAGAGTT \\
\hline \multicolumn{2}{|c|}{ phaCF1 ..............................TAGGATCCATGTCCAGCAACCCCTTC } \\
\hline \multicolumn{2}{|c|}{ phaCR1 ..............................GTGGTACCTTACAGTTGATCGAGCCA } \\
\hline \multicolumn{2}{|c|}{ phaCF2 ...........................TTAGGATCCTCCAGCAАССССTTCAAT } \\
\hline \multicolumn{2}{|c|}{ phaCR2 ...........................GCGGAATTCTTACAGTTGATCGAGCCA } \\
\hline \multicolumn{2}{|c|}{ phaEF1 .............................TTGGATCCCAGCTCGAAGAAGTGCAG } \\
\hline \multicolumn{2}{|c|}{ phaER1 ...........................GGCGGTACCTTATTCTTCTAAGTGTTC } \\
\hline \multicolumn{2}{|c|}{ phaEF2 ..............................GGGGATCCATGAGTAATACAAACAAC } \\
\hline \multicolumn{2}{|c|}{ phaER2 ............................... GGAATTCTATTCTTCTAAGTGTTC } \\
\hline \multicolumn{2}{|c|}{ RTEF ...............................ACATGAAGGCCCAGGCGG } \\
\hline \multicolumn{2}{|c|}{ RTER ................................CGTCCGCTTCCTGTTGCA } \\
\hline \multicolumn{2}{|c|}{ RTECF ...........................GGAAGCGGACGACCTGAG } \\
\hline \multicolumn{2}{|c|}{ RTECR.............................CGGTTGATGAGCGCGTAA } \\
\hline \multicolumn{2}{|l|}{ RTCF ....... } \\
\hline \multicolumn{2}{|c|}{ RTCR .............................TCGAGGACGCCGCCAGTGTG } \\
\hline TIP ......... & ......СТССТСААССАТСТССGTCC \\
\hline \multicolumn{2}{|r|}{........ CACGACTCCACGAAGGCCGC } \\
\hline \multicolumn{2}{|c|}{ CrossF1 ..............................GAAAGCTTCCAGCAAAACGTCAACAG } \\
\hline \multicolumn{2}{|c|}{ CrossR1 .............................TATGGATCCCTTCGGCCTGCACTTCCT } \\
\hline CrossF2. & ......TATGGATCCCTCGAAGACGTGTATCAGGAC \\
\hline CrossR2 & .....TATGGTACCCGGGATTCGGTGGTTTCG \\
\hline
\end{tabular}

${ }^{a}$ Sequences representing restriction sites are underlined. 

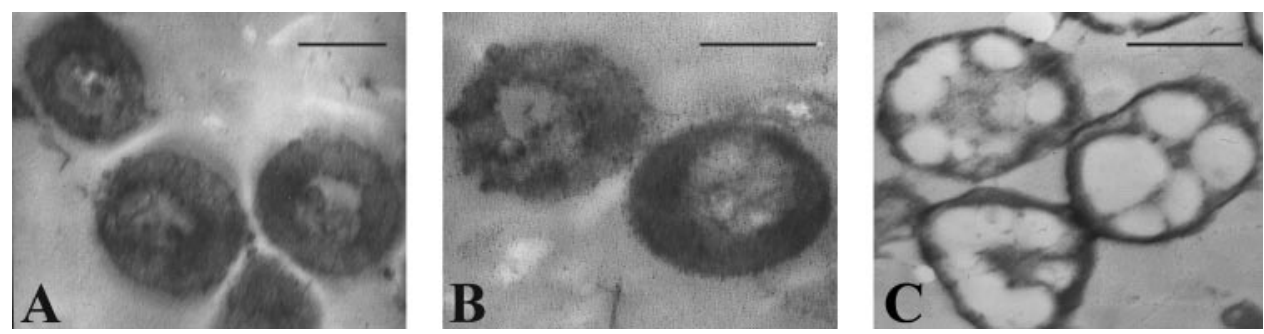

FIG. 1. TEM images of $H$. marismortui ATCC 43049 cells grown at $37^{\circ} \mathrm{C}$ for $72 \mathrm{~h}$ in different media: (A) nutrient-rich AS-168 medium; (B) minimal medium supplemented with $2 \%$ glucose and $0.5 \%$ Casamino Acids (MGC medium); and (C) minimal medium supplemented with $2 \%$ glucose (MG medium). Bar, $0.5 \mu \mathrm{m}$.

pUBP2 with the pHH9-ori region omitted by EcoRI digestion, resulting in the integration plasmid pUBPHL (Table 1). Transformation of pUBPHL into ATCC 33960 and screening of a phaEC $C_{\mathrm{Hh}}$ deletion mutant, named $H$. hispanica PHB-1, were carried out according to the method of Tu et al. (39). The plasmids pWLE, pWLfdxC, and pWLEC were transformed into $H$. hispanica PHB-1 to verify the functions of $p h a E C_{\mathrm{Hm}}$ genes.

These haloarchaeal plasmids were usually constructed in E. coli JM109 at first and then transformed into $H$. volcanii and $H$. hispanica with the polyethylene glycol-mediated transformation method described by Cline et al. (4). The PCRamplified sequence in each construct was verified by DNA sequencing.

Isolation and characterization of PHA granules. Haloarchaeal cells accumulating PHA were harvested by centrifugation and washed once with basal salt solution (BSS; per liter, $200 \mathrm{~g} \mathrm{NaCl}, 20 \mathrm{~g} \mathrm{MgSO}_{4} \cdot 7 \mathrm{H}_{2} \mathrm{O}, 2 \mathrm{~g} \mathrm{KCl}$, and $3 \mathrm{~g}$ trisodium citrate). Cellular PHA content and its composition were analyzed by gas chromatography (GC). Briefly, the lyophilized cells were subjected to methanolysis in a mixture of chloroform and methanol containing 3\% (vol/ $/ \mathrm{vol})$ sulfuric acid at $100^{\circ} \mathrm{C}$ for $4 \mathrm{~h}$. The resulting hydroxyacyl methylesters were then analyzed with a GC-6820 apparatus (Agilent). The PHB content (as percent weight/weight) in the cells was calculated as follows: (mass of PHB/original lyophilized cell mass) $\times 100 \%$.

For isolation of PHA granules, PHA-accumulating cells were centrifuged, washed once with BSS, and resuspended in a solution containing $20 \mathrm{mM}$ Tris$\mathrm{HCl}(\mathrm{pH} 7.5), 200 \mathrm{~g} /$ liter $\mathrm{NaCl}, 20 \mathrm{~g} /$ liter $\mathrm{KCl}$, and $5 \mathrm{~g} /$ liter $\mathrm{MgSO}_{4} \cdot 7 \mathrm{H}_{2} \mathrm{O}$. Crude extracts were obtained by passing cells three times through a French press $\left(13,000 \mathrm{lb} / \mathrm{in}^{2}\right.$; Thermo IEC). Intact cells and debris were pelleted by centrifugation $\left(10,000 \times g, 4^{\circ} \mathrm{C}, 30 \mathrm{~min}\right)$. PHA granules in the supernatants were collected by ultracentrifugation $\left(200,000 \times g, 4^{\circ} \mathrm{C}, 2 \mathrm{~h}\right)$ and then suspended in the same buffer and stored at $-70^{\circ} \mathrm{C}$ for future use.

TEM. H. marismortui ATCC 43049 cells cultivated in AS-168, MG, or MGC medium (MG medium plus $0.5 \%$ Casamino Acids) for $72 \mathrm{~h}$ were harvested by centrifugation, washed twice with BSS, and then subjected for transmission electron microscopic (TEM) analysis as described by Pötter et al. (28). Micrographs were recorded with a Hitachi H-700A electron microscopy.

Reverse transcriptase PCR and primer extension. Total RNA of H. marismortui ATCC 43049 was isolated with the TRIzol reagent (Gibco BRL) from cells cultured for $72 \mathrm{~h}$. Prior to the reverse transcription (RT) reaction, about 10 $\mu \mathrm{g}$ of total RNA was treated with RNase-free RQ1 DNase (Promega) to eliminate any contamination of DNA. The DNA-free RNA was used for cDNA synthesis with SuperScript III reverse transcriptase (Invitrogen) using the antisense primer RTCR (Table 2). After incubation for $1 \mathrm{~h}$ at $42^{\circ} \mathrm{C}$, the RT reaction was terminated by incubation at $70^{\circ} \mathrm{C}$ for $20 \mathrm{~min}$, and $1 \mu \mathrm{l}$ of reverse transcripts was applied for PCR with the primer pair RTEF/RTER for the $p h a E_{\mathrm{Hm}}$ gene, RTCF/RTCR for $p h a C_{\mathrm{Hm}}$, and RTECF/RTECR for $p h a E C_{\mathrm{Hm}}$ (Table 2). These RT-PCR products were separated in $1 \%$ agarose gel, followed by ethidium bromide staining.

For determination of the transcriptional start site of $p h a E C_{\mathrm{Hm}}$ genes, total RNA was obtained from the $H$. volcanii transformants harboring pWLEC or pWL102. The RNA samples $(6 \mu \mathrm{g})$ were used as templates for primer extensions, while the PCR product amplified with primer pair phaEF1/seqR was used as template for DNA sequencing. The primer TIP (Table 1) was labeled at the $5^{\prime}$ end with $\left[\gamma_{-}{ }^{32} \mathrm{P}\right] \mathrm{ATP}$ and was used for both DNA sequencing and primer extension as described previously (42).

Expression and purification of $\mathrm{PhaE}_{\mathrm{Hm}}-\mathrm{His}_{6}$ and $\mathrm{PhaC}_{\mathrm{Hm}}-\mathrm{His}_{6}$ in E. coli. Plasmids of pET28aE and pET28aC were constructed to express $\mathrm{PhaE}_{\mathrm{Hm}}-\mathrm{His}_{6}$ and $\mathrm{PhaC}_{\mathrm{Hm}^{-}}-\mathrm{His}_{6}$ in E. coli BL21(DE3). The E. coli recombinants were cultured until the mid-exponential phase and then induced with isopropyl- $\beta$-D-thiogalactopyranoside (IPTG; $1 \mathrm{mM}$ ) for an additional $4 \mathrm{~h}$. The His-tagged proteins were purified using the His-bind column (Novagen) according to the manufacturer's instructions. The concentrations of proteins were determined with the bicinchoninic acid protein assay kit (Pierce).

Antisera preparation and Western blot analysis. The antisera were prepared by immunizing mice with purified $\mathrm{PhaE}_{\mathrm{Hm}}-\mathrm{His}_{6}$ and $\mathrm{PhaC}_{\mathrm{Hm}}-\mathrm{His}_{6}$ as previously described (37). For Western blot analysis, crude extracts from H. marismortui ATCC 43049 were obtained by disrupting cells through a French press as described above. Soluble fractions were obtained by centrifugation at $10,000 \times g$ for $30 \mathrm{~min}$. One hundred micrograms of proteins from cellular extracts and PHA granules were separated on $12 \%$ sodium dodecyl sulfate-polyacrylamide gels, and 0.5- $\mu$ g aliquots of purified $\mathrm{PhaE}_{\mathrm{Hm}}-\mathrm{His}_{6}$ and $\mathrm{PhaC}_{\mathrm{Hm}}-\mathrm{His}_{6}$ proteins were used as controls. The proteins on the gel were then transferred onto a polyvinylidene difluoride membrane by using a semidry electrophoretic transfer cell (Bio-Rad) at $15 \mathrm{~V}$ for $15 \mathrm{~min}$. The blots were blocked in $7 \%$ nonfat dry milk in phosphate-buffered saline ( $\mathrm{pH}$ 7.4) for $30 \mathrm{~min}$ and then incubated with the anti-PhaE $\mathrm{Hm}_{-}-\mathrm{His}_{6}$ or anti-PhaC $\mathrm{Hm}^{-}-\mathrm{His}_{6}$ antiserum for $3 \mathrm{~h}$. After appropriate washing, the blots were incubated with alkaline phosphatase-labeled goat antimouse immunoglobulin $\mathrm{G}$ for $30 \mathrm{~min}$ at room temperature, followed by staining in the alkaline phosphatase development solution containing nitroblue tetrazolium and 5-bromo-4-chloro-3-indolylphosphate.

PHA synthase activity assay. PHA synthase activity was measured spectrophotometrically by recording the release of $\mathrm{CoA}$ during the polymerization of 3-hydroxybutyryl-CoA (3HB-CoA). The assay mixture (1 ml) contained $20 \mathrm{mM}$ Tris- $\mathrm{HCl}$ (pH 7.5), $3.4 \mathrm{M} \mathrm{KCl}, 100 \mu \mathrm{M} \mathrm{Mg}\left(\mathrm{CH}_{3} \mathrm{COO}\right)_{2}, 100 \mu \mathrm{M}$ 3HB-CoA, 1 $\mathrm{g} /$ liter bovine serum albumin, and $500 \mu \mathrm{g}$ of protein extracts. The reaction was initiated by the addition of PHA synthase samples at $30^{\circ} \mathrm{C}$. The absorbance at $412 \mathrm{~nm}$ was measured at defined time points. The concentration of CoA was determined using a molar absorption coefficient of $13,600 \mathrm{M}^{-1} \mathrm{~cm}^{-1}$ (5). One unit was defined as the amount of enzyme that catalyzed the generation of 1 $\mu \mathrm{mol} \mathrm{CoA} \mathrm{per} \mathrm{min.}$

Nucleotide sequence accession number. According to the DNA sequence of phaE $C_{\mathrm{Hm}}$ of $H$. marismortui ATCC 43049 (GenBank accession no. AY596297), the highly homologous $p h a E C_{\mathrm{Hh}}$ genes of $H$. hispanica ATCC 33960 were cloned and sequenced and deposited in GenBank under the accession number EU022705.

\section{RESULTS}

PHA accumulation in $\boldsymbol{H}$. marismortui under nutrient-limited conditions. The capability for PHA synthesis in $H$. marismortui ATCC 43049 was investigated under different culture conditions (see Materials and Methods). Although almost no PHA synthesis was observed when this haloarchaeal strain was cultivated in nutrient-rich medium AS-168 (Fig. 1A) or minimal medium with Casamino Acids and glucose (MGC medium, $\mathrm{pH}$ 7.2) (Fig. 1B), large amounts of PHA-insoluble granules were detected in the cells when cultured in minimal medium with $2 \%$ glucose as the excessive carbon source ( $\mathrm{MG}$ medium, $\mathrm{pH}$ 7.2) (Fig. 1C). The typical PHA granules contributed a significant fraction of the cell volume, with similar shape and size ( 0.1 to $0.5 \mu \mathrm{m}$ in diameter) as those in bacteria. These observations were confirmed by GC analysis, with which the PHA granules were further identified to be PHB. 


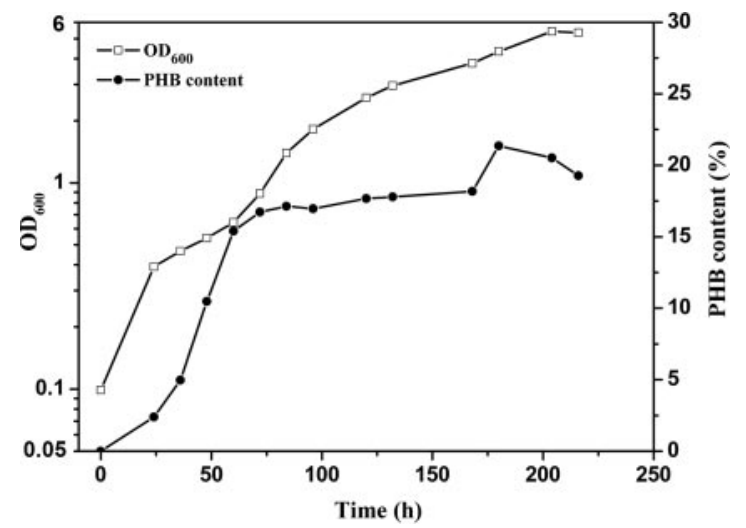

FIG. 2. Profiles of PHB accumulation and cell growth of $H$. marismortui ATCC 43049 cultivated in MG medium in a 5-liter fermentor (NBS Bioflo 110 ) at $37^{\circ} \mathrm{C}$ for about 10 days. The PHB content and cell density, as the optical density at $600 \mathrm{~nm}\left(\mathrm{OD}_{600}\right)$, were monitored. This figure represents a typical result of three independent experiments.

To investigate the relationship between cell growth and PHB content in H. marismortui ATCC 43049 when cultured in MG medium, the time profile of the PHB accumulation was determined (Fig. 2). The PHB content increased quickly in the first $72 \mathrm{~h}$ and remained relative stable, at about $17 \%(\mathrm{wt} / \mathrm{wt})$ of cellular dry weight from $72 \mathrm{~h}$ to $168 \mathrm{~h}$. The PHB content reached its maximum at $21 \%$ (wt/wt) after $180 \mathrm{~h}$ of fermentation and then began to decrease slowly (Fig. 2). This was a typical result of several independent investigations. Thus, in the following experiments when we analyzed gene expression and PHA synthase activity as well as PHB accumulation, we usually collected the cells at 72 to $\sim 180 \mathrm{~h}$ of fermentation, corresponding to an atypical exponential phase. It is notable that some other factors, including concentration of nitrogen and phosphorus as well as the oxygen limitation that might affect the PHB accumulation, were not investigated in this study.

Identification of phaEC $_{\mathrm{Hm}}$ genes for PHB synthesis in $\boldsymbol{H}$. marismortui. The genome sequence of $H$. marismortui ATCC 43049 has been published recently (1). In this genome there is an open reading frame (ORF) annotated as the $p h a C_{\mathrm{Hm}}$ gene, which encodes a 475-amino-acid protein. $\mathrm{PhaC}_{\mathrm{Hm}}$ contains a highly conserved "lipase box-like" sequence (Gly-X-Cys-XGly-Gly) (Fig. 3A), which is believed to be an active site of PHA synthase (7). Moreover, the amino acids Cys162-Asp317His346, as equivalents of the catalytic triad residues Cys149Asp303-His331 in $\mathrm{PhaC}_{\mathrm{Av}}(12,27)$, were also detected in this protein. Significantly, $\mathrm{PhaC}_{\mathrm{Hm}}$ showed high homology (37 to $43 \%$ identical amino acids) with the PhaC subunits of the bacterial class III PHA synthases and contained the conserved motif Arg-Met-Glu-X-Trp-Ile-X-Asp-X-X-Asp (Fig. 3A) that is typically found in class III synthases (9). These results suggested that $\mathrm{PhaC}_{\mathrm{Hm}}$ might be one subunit of the class III PHA synthase in $H$. marismortui. Interestingly, immediately upstream of phaC $C_{\mathrm{Hm}}$ gene, we detected another ORF with unknown function (Fig. 4A) which encoded a protein showing sequence similarity (21 to $25 \%$ identical amino acids) with the PhaE subunits of class III PHA synthases in bacteria (8) and contained a typical "PhaE box" (Fig. 3B). Therefore, we designated this ORF as phaE $E_{\mathrm{Hm}}$ gene and propose that the
phaE $C_{\mathrm{Hm}}$ genes encode two subunits of a functional PHA synthase in $H$. marismortui.

Cotranscription of phaEC $C_{\mathrm{Hm}}$ genes from the same promoter. By analysis of the upstream sequence of the $p h a E C_{\mathrm{Hm}}$ genes, we detected a putative archaeal TATA box (TTTTTTT) and a transcription factor B recognition element (GG), which might be the promoter controlling the transcription of both $p h a E_{\mathrm{Hm}}$ and pha $C_{\mathrm{Hm}}$, as there was only a 2-base spacing between these two genes (Fig. 4A). Primer extension analysis revealed that the transcription start point of the $p h a E_{\mathrm{Hm}}$ gene was located 26 bp downstream of this putative promoter, exactly at base A of the start codon (AUG) of the pha $E_{\mathrm{Hm}}$ gene (Fig. 4B). Thus, it was another example of a "leaderless" transcript in haloarchaea (31).

To determine whether $p h a E_{\mathrm{Hm}}$ and $p h a C_{\mathrm{Hm}}$ were cotranscribed under the same promoter, RT-PCR was performed to check the existence of a polycistronic transcript of phaEC $C_{\mathrm{Hm}}$. Indeed, the PCR products (F1, F2, and F3) corresponding to the partial sequences of $p h a E_{\mathrm{Hm}}, p h a C_{\mathrm{Hm}}$, and $p h a E C_{\mathrm{Hm}}$ mRNA with expected lengths were generated by using the primer pairs RTEF/RTER, RTCF/RTCR, and RTECF/ RTECR (Table 1), respectively (Fig. 4C). These results indicated that $p h a E_{\mathrm{Hm}}$ and $p h a C_{\mathrm{Hm}}$ were cotranscribed and were constitutively expressed no matter whether under the PHA accumulation condition (e.g., in MG medium) or not (e.g., in AS-168 medium), which was also confirmed by Northern blot analysis (data not shown).

Western blot analysis of $\mathrm{PhaE}_{\mathrm{Hm}}$ and $\mathrm{PhaC}_{\mathrm{Hm}}$ proteins. To reveal the levels of $\mathrm{PhaE}_{\mathrm{Hm}}$ and $\mathrm{PhaC}_{\mathrm{Hm}}$ and their relationship with PHB synthesis in H. marismortui ATCC 43049, Western blotting was performed to detect the production of $\mathrm{PhaC}_{\mathrm{Hm}}$ and $\mathrm{PhaE}_{\mathrm{Hm}}$ under different culture conditions. Both $\mathrm{PhaC}_{\mathrm{Hm}}$ and $\mathrm{PhaE}_{\mathrm{Hm}}$ were detected in the cellular extracts (Fig. 5), but interestingly, while a much stronger signal of $\mathrm{PhaC}_{\mathrm{Hm}}$ was detected from the PHA granules (Fig. 5B), no signal of $\mathrm{PhaE}_{\mathrm{Hm}}$ was detected (Fig. 5A). This suggested that $\mathrm{PhaC}_{\mathrm{Hm}}$ was stably attached to the PHA granules while $\mathrm{PhaE}_{\mathrm{Hm}}$ might not or just weakly combine with the PHA granules. Thus, $\mathrm{PhaE}_{\mathrm{Hm}}$ and $\mathrm{PhaC}_{\mathrm{Hm}}$ likely constitute, if anything, a novel form of class III PHA synthases in H. marismortui.

In accordance with the RT-PCR results (Fig. 4C), both proteins $\left(\mathrm{PhaE}_{\mathrm{Hm}}\right.$ and $\left.\mathrm{PhaC}_{\mathrm{Hm}}\right)$ in the crude extracts of cells that were cultivated in either AS-168 or MG medium showed a similar amount in Western blot analysis (Fig. 5), suggesting that $\mathrm{PhaE}_{\mathrm{Hm}}$ and $\mathrm{PhaC}_{\mathrm{Hm}}$ are indeed constitutively expressed. We also found that the PHA synthase activity of $H$. marismortui ATCC 43049 was similar when grown in both media, indicating that the activity of the PHA synthase was also not regulated at the protein level (data not shown). This constitutive expression of the putative PHA synthase was different from that of the halophilic archaeon strain 56 (11) but has been widely observed in bacteria $(7,16,33)$.

Overexpression of $\mathrm{PhaE}_{\mathrm{Hm}}$ and $\mathrm{PhaC}_{\mathrm{Hm}}$ in $\mathrm{H}$. hispanica. To demonstrate the function of phaEC $C_{\mathrm{Hm}}$ genes, the DNA fragments containing phaE $E_{\mathrm{Hm}}, p h a C_{\mathrm{Hm}}$, and phaEC $C_{\mathrm{Hm}}$ were inserted into pWL102, resulting in pWLE, pWLfdxC, and pWLEC, respectively (see Materials and Methods). These plasmids were introduced into $H$. hispanica ATCC 33960, a haloarchaeon that is phylogenetically close to $H$. marismortui ATCC 43049 and is much more easily transformed. 

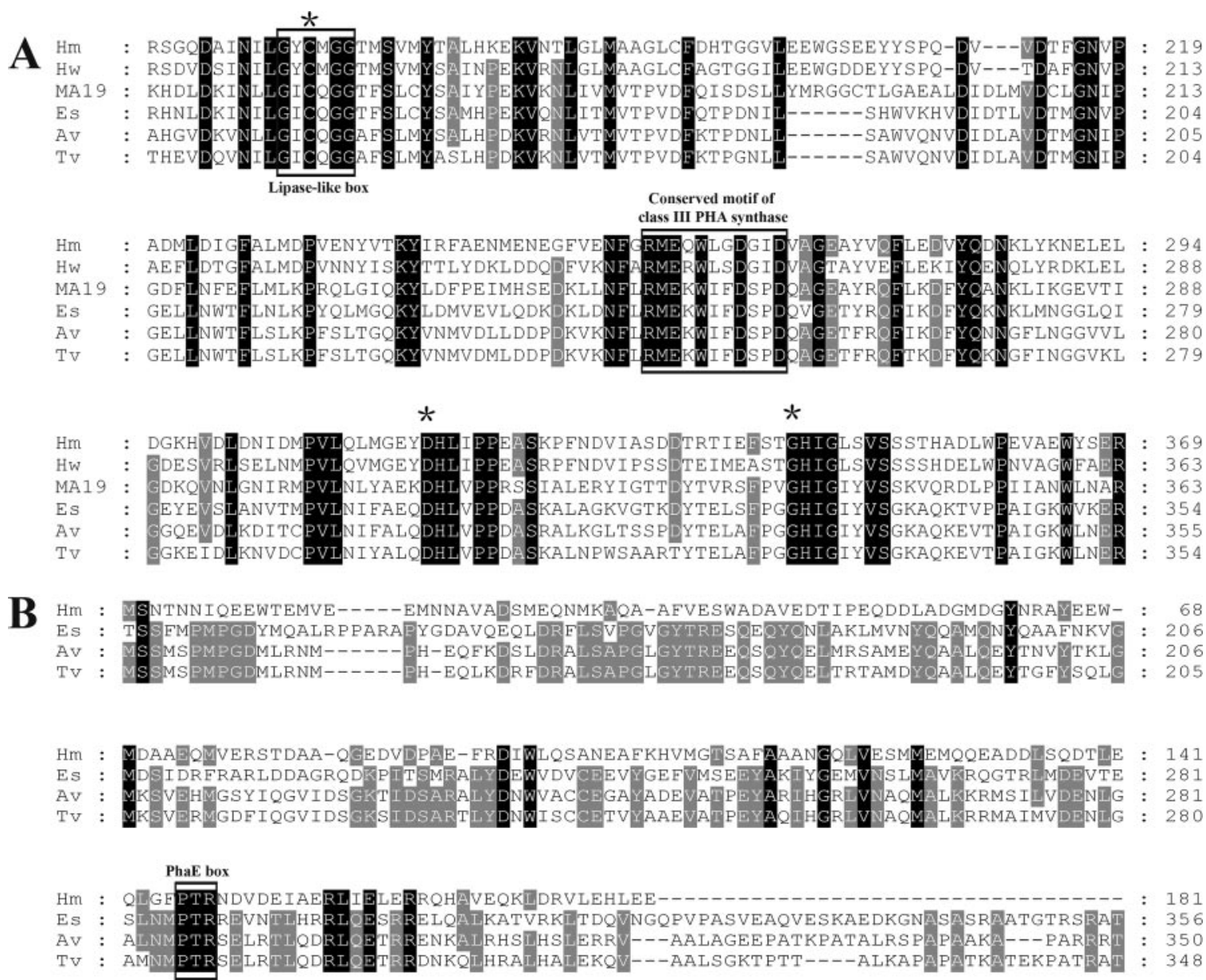

FIG. 3. Partial alignments of the amino acid sequences of PhaC (A) and PhaE (B) subunits from H. marismortui ATCC 43049 (Hm), H. walsbyi DSM 16790 (Hw), Synechococcus sp. strain MA19 (MA19), Ectothiorhodospira shaposhnikovii (Es), Allochromatium vinosum (Av), and Thiocystis violacea $(\mathrm{Tv})$. Amino acids are given in standard one-letter abbreviations, and the numbers indicate the positions of the amino acids within the respective proteins. The conserved residues are darkly shaded, and the residues identical in five of the six (for PhaC) or three of the four (for PhaE) are lightly shaded. The lipase-like box, highly conserved motif of class III synthase, and PhaE box are indicated. The conserved catalytic triad residues are shown with asterisks. GenBank accession numbers are as follows: $\mathrm{PhaC}_{\mathrm{Hm}}, \mathrm{YP}_{\mathbf{A}} 137339 ; \mathrm{PhaC}_{\mathrm{Hw}}, \mathrm{YP}_{\mathbf{P}} 658052 ; \mathrm{PhaC}_{\mathrm{MA19}}, \mathrm{AAK38139}$;

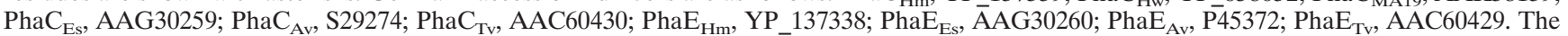
corresponding PhaE sequences were not identified in H. walsbyi DSM 16790 and Synechococcus sp. strain MA19.

H. hispanica ATCC 33960 harbors a highly homologous phaEC $C_{\mathrm{Hh}}$ operon (GenBank accession no. EU022705), which exhibits more than $92 \%$ identity with $p h a E C_{\mathrm{Hm}}$. As expected, overexpression of $p h a E_{\mathrm{Hm}}$ and/or phaC $C_{\mathrm{Hm}}$ in ATCC 33960 promoted $\mathrm{PHB}$ accumulation in all of the recombinant cells, e.g., at $144 \mathrm{~h}$, the PHB content in the cells could be increased from $9.9 \%$ (wt/wt) to up to 16.0 to $18.0 \%$ (wt/wt) (Table 3). Interestingly, overexpression of $p h a E_{\mathrm{Hm}}$ or $p h a C_{\mathrm{Hm}}$ did not affect the biomass, while coexpression of phaEC $C_{\mathrm{Hm}}$ genes slightly promoted the cellular dry weight, which was increased from $12.4 \mathrm{~g} /$ liter (for 33960/pWL102) to $15.4 \mathrm{~g} /$ liter (for 33960/ pWLEC) after $144 \mathrm{~h}$ of fermentation (Table 3). Thus, the PHB yields in 33960/pWLE and 33960/pWLfdxC were, respectively, increased up to $167 \%(\sim 2.0 \mathrm{~g} /$ liter $)$ and $175 \%(\sim 2.1 \mathrm{~g} /$ liter $)$ of that in vector-transformed cells $(\sim 1.2 \mathrm{~g} /$ liter $)$ and, significantly, coexpression of $p h a E C_{\mathrm{Hm}}$ had the most significant effect on PHB synthesis. The PHB yield in 33960/pWLEC increased up to $233 \%$ ( $\sim 2.8 \mathrm{~g} /$ liter $)$ of that in the control cells after $144 \mathrm{~h}$ of fermentation (Table 3). These results suggested that both phaE and phaC genes were involved in PHA synthesis in the Haloarcula species.

Both phaE and phaC genes are necessary for an active PHA synthase in Haloarcula. To further clarify the functions of the phaEC $C_{\mathrm{Hm}}$ genes, the PHA synthase activities in the phaEC $\mathrm{Hm}^{-}$ overexpressing $H$. hispanica strains were assayed. Compared with that in the control strain $H$. hispanica ATCC 33960/ pWL102 ( $\sim 1.0 \mathrm{U} / \mathrm{mg}$ protein), it was revealed that the PHA synthase activities of the crude extracts from $H$. hispanica recombinants harboring phaE ${ }_{\mathrm{Hm}}(33960 / \mathrm{pWLE} ; \sim 2.0 \mathrm{U} / \mathrm{mg}$ protein) or $p h a C_{\mathrm{Hm}}(33960 / \mathrm{pWLfdxC} ; \sim 1.6 \mathrm{U} / \mathrm{mg}$ protein) were clearly increased. Again, coexpression of phaE $C_{\mathrm{Hm}}$ genes in $H$. hispanica (33960/pWLEC) resulted in the highest PHA synthase activity $(\sim 2.6 \mathrm{U} / \mathrm{mg}$ protein $)$. These results suggested that the phaEC genes might encode the same subunits of PHA synthase in Haloarcula as those observed in bacteria. To confirm this, we knocked out the $p h a E C_{\mathrm{Hh}}$ genes from $H$. his- 


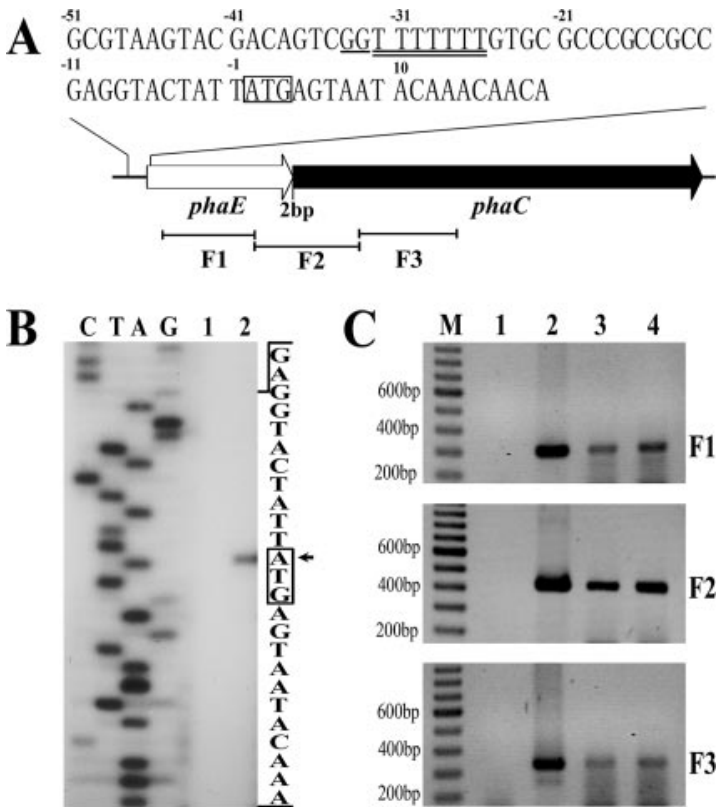

FIG. 4. Transcription analysis of phaE $C_{\mathrm{Hm}}$ genes. (A) Structure and organization of the phaEC $C_{\mathrm{Hm}}$ genes in H. marismortui. The putative promoter containing a TATA box (double underlined) and a transcription factor $\mathrm{B}$ recognition element (single underlined) and the translational start codon of $p h a E_{\mathrm{Hm}}$ (boxed) are indicated. F1, $\mathrm{F} 2$, and $\mathrm{F} 3$ represent the locations of the RT-PCR products (shown in panel C) in the phaEC $C_{\mathrm{Hm}}$ genes. (B) Mapping of the transcriptional start site of phaEC $C_{\mathrm{Hm}}$ by primer extension. The relevant sequence is shown on the right. The transcriptional start site is indicated with an arrow. Lane $1, \mathrm{H}$. volcanii/pWL102, negative control; lane $2, \mathrm{H}$. volcanii/pWLEC; lanes CTAG, standard sequencing reaction mixture to size the mapping signals. (C) RT-PCR products of partial $p h a E_{\mathrm{Hm}}(\mathrm{F} 1$; $314 \mathrm{bp}$ ), phaEC $C_{\mathrm{Hm}}(\mathrm{F} 2 ; 434 \mathrm{bp})$, and pha $C_{\mathrm{Hm}}$ (F3; $\left.341 \mathrm{bp}\right)$ sequences. The following templates were used in the PCR: lane 1, total RNA without reverse transcriptase; lane 2, H. marismortui genomic DNA; lane 3, reverse transcripts of total RNA isolated from cells grown in AS-168 medium; lane 4, reverse transcripts of total RNA isolated from cells grown in MG medium; lane M, DNA marker.

panica ATCC 33960 (see Materials and Methods) and thus generated a phaEC-deleted strain named $H$. hispanica PHB-1. As expected, $H$. hispanica PHB-1 completely lost the PHA synthase activity and the capability for PHB synthesis when cultivated in MG medium. This result supported the argument that the phaEC genes might encode the PHA synthase in the Haloarcula species. To further evaluate the function of PhaE and $\mathrm{PhaC}$ in PHB synthesis, the plasmids pWLE, pWLfdxC, and $\mathrm{pWLEC}$ were also transformed into $H$. hispanica PHB-1. It was revealed that neither pWLE nor pWLfdxC, but only

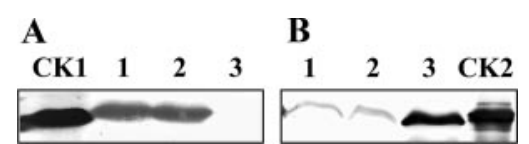

FIG. 5. Western blot analysis of cellular extracts and PHA granules from $H$. marismortui ATCC 43049 with antiserum against $\mathrm{PhaE}_{\mathrm{Hm}}$ (A) or $\mathrm{PhaC}_{\mathrm{Hm}}$ (B). Lane 1, crude extracts from cells grown in AS-168 medium; lane 2, crude extracts from cells grown in MG medium; lane 3 , proteins from isolated PHA granules. The purified $\mathrm{PhaE}_{\mathrm{Hm}}-\mathrm{His}_{6}$ (CK1) and $\mathrm{PhaC}_{\mathrm{Hm}^{-}}-\mathrm{His}_{6}$ (CK2) from $E$. coli were used as controls. $H$. marismortui ATCC 43049 was cultivated at $37^{\circ} \mathrm{C}$ for $72 \mathrm{~h}$.
TABLE 3. PHB accumulation in H. hispanica ATCC 33960 recombinant strains

\begin{tabular}{|c|c|c|c|c|}
\hline \multirow{3}{*}{ Strain } & \multicolumn{4}{|c|}{ PHB accumulation after: } \\
\hline & \multicolumn{2}{|c|}{$96 \mathrm{~h}$} & \multicolumn{2}{|c|}{$144 \mathrm{~h}$} \\
\hline & $\begin{array}{l}\text { CDW } \\
\text { (g/liter) }\end{array}$ & $\begin{array}{l}\text { PHB/CDW } \\
(\%[w t / w t])\end{array}$ & $\begin{array}{l}\text { CDW } \\
\text { (g/liter) }\end{array}$ & $\begin{array}{l}\text { PHB/CDW } \\
(\%[w t / w t])\end{array}$ \\
\hline 33960/pWL102 & 11.2 & 8.3 & 12.4 & 9.9 \\
\hline 33960/pWLE & 11.5 & 11.3 & 12.6 & 15.8 \\
\hline 33960/pWLfdxC & 11.3 & 11.2 & 12.3 & 16.7 \\
\hline 33960/pWLEC & 13.6 & 14.6 & 15.4 & 18.0 \\
\hline
\end{tabular}

pWLEC, could restore the PHA synthase activity (data not shown). Consistently, only coexpression of $p h a E C_{\mathrm{Hm}}$ genes in H. hispanica PHB-1 restored the ability to accumulate PHB (Fig. 6C). Expression of either pha $E_{\mathrm{Hm}}$ or $p h a C_{\mathrm{Hm}}$ in $H$. hispanica PHB-1 did not result in PHB accumulation (Fig. 6A and B). These results strongly suggested that the phaEC genes encode two subunits, PhaE and PhaC, of a novel class III PHA synthase in the Haloarcula species.
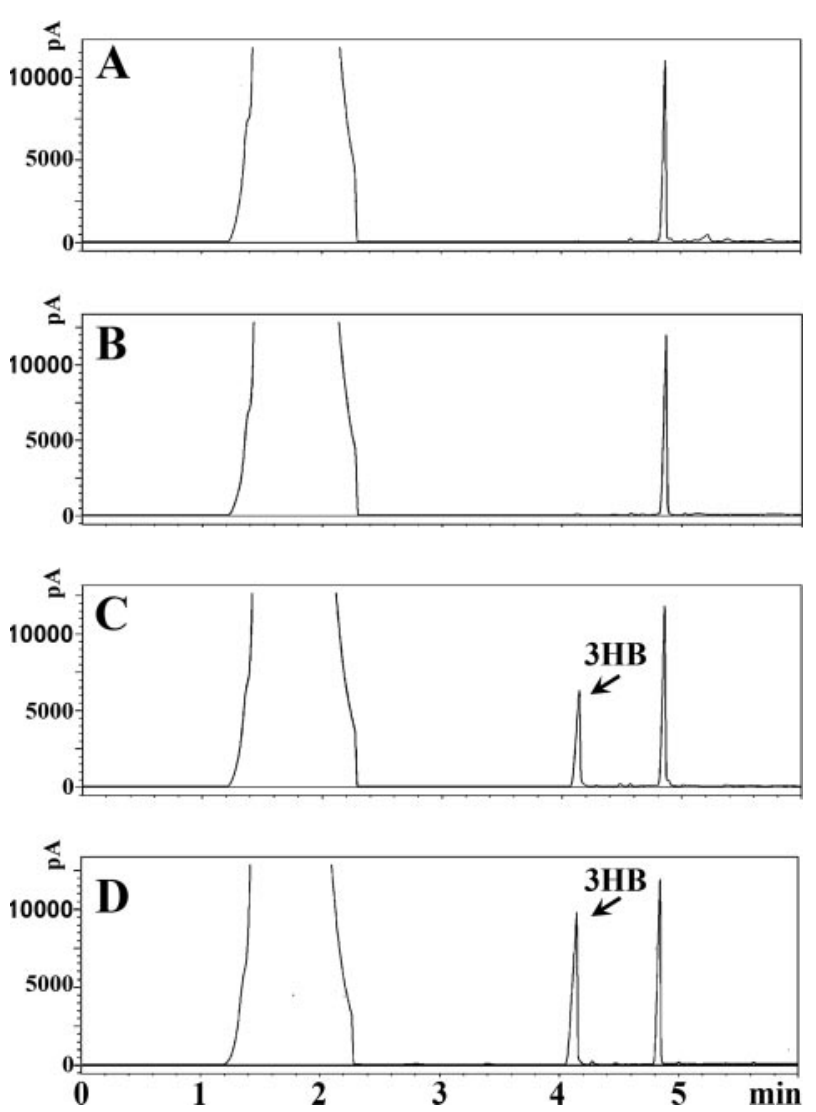

FIG. 6. GC analysis of PHB accumulation in $H$. hispanica PHB-1 recombinants. The $3 \mathrm{HB}$ peaks are the actual peaks of 3-hydroxybutyrate methylester, the methanolysis product of PHB (see Materials and Methods). (A) $H$. hispanica PHB-1 harboring pWLE; (B) $H$. hispanica PHB-1 harboring pWLfdxC; (C) H. hispanica PHB-1 harboring pWLEC; (D) PHB standard (Sigma). The peak at 4.85 min represents the methylester product of an internal standard (1 ng of benzoic acid). The $H$. hispanica PHB-1 recombinants were cultivated in $\mathrm{MG}$ medium at $37^{\circ} \mathrm{C}$ for $96 \mathrm{~h}$. 


\section{DISCUSSION}

In the present study, we report for the first time the identification and molecular characterization of two genes, phaE $E_{\mathrm{Hm}}$ and $p h a C_{\mathrm{Hm}}$, that are essential for both $\mathrm{PHB}$ accumulation and PHA synthase activity in H. marismortui ATCC 43049. We have determined their genetic organization, studied their regulation at the transcriptional and translational level, and proved by synthase activity assay and PHB accumulation analysis in a heterologous system as well as a gene knockout/ complementation system that the phaEC genes might encode two subunits of an active PHA synthase in Haloarcula.

It is noteworthy that the PHA synthase in H. marismortui was likely a homolog of the bacterial class III synthase, as both $\mathrm{PhaE}_{\mathrm{Hm}}$ and $\mathrm{PhaC}_{\mathrm{Hm}}$ contained conserved motifs typically found in class III PHA synthases from Bacteria (Fig. 3). Sim-

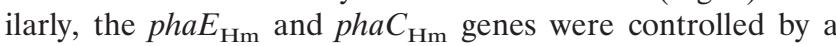
single promoter (Fig. 4), which also suggested that $\mathrm{PhaE}_{\mathrm{Hm}}$ and $\mathrm{PhaC}_{\mathrm{Hm}}$ might function together in $H$. marismortui. However, the protein sizes of $\mathrm{PhaE}_{\mathrm{Hm}}(20.64 \mathrm{kDa})$ and $\mathrm{PhaC}_{\mathrm{Hm}}$ $(53.12 \mathrm{kDa})$ were distinct from typical class III PHA synthases of bacteria. e.g., the $\mathrm{PhaE}_{\mathrm{Av}}$ and $\mathrm{PhaC}_{\mathrm{Av}}$ of $A$. vinosum were about $40.53 \mathrm{kDa}$ and $39.73 \mathrm{kDa}$, respectively (22). Moreover, the stretch rich in hydrophobic and amphiphilic amino acids for granule association in most bacterial PhaE subunits (20) was absent from the $\mathrm{C}$ terminal of $\mathrm{PhaE}_{\mathrm{Hm}}$. These results indicated that the PHA synthase of $H$. marismortui was a novel form and may explain why Western blotting could only detect $\mathrm{PhaC}_{\mathrm{Hm}}$ but not $\mathrm{PhaE}_{\mathrm{Hm}}$ associated with $\mathrm{PHB}$ granules (Fig. 5). It is possible that $\mathrm{PhaE}_{\mathrm{Hm}}$ combined with $\mathrm{PhaC}_{\mathrm{Hm}}$ with a weak strength to form an active complex enzyme, and the conditions of purifying the PHB granules might have released $\mathrm{PhaE}_{\mathrm{Hm}}$. Besides PhaE and PhaC from the genus Haloarcula, the $\mathrm{PhaC}_{\mathrm{Hw}}$ subunit from another genus of haloarchaea, $H$. walsbyi DSM 16790, also showed high homology with the class III PhaC (Fig. 3), thereby indicating that the class III PHA synthases might be widely spread from Bacteria to Archaea.

H. marismortui ATCC 43049 accumulated PHB when it was cultured in minimal medium ( $\mathrm{pH}$ 7.2) with excess glucose (Fig. $1)$. But, surprisingly, we revealed that the $p h a E C_{\mathrm{Hm}}$ genes were constitutively expressed, no matter whether the cells were cultured under nutrient-rich (AS-168 medium) or nutrient-limited (MG medium) conditions (Fig. 4 and 5). This was different from results with the haloarchaeal strain 56, whose PHA synthase activity presented only under PHB-accumulating conditions (11), but resembled many bacteria, including Cupriavidus necator, A. vinosum, Synechococcus sp., and Paracoccus denitrificans, whose PHA synthases are also constitutively expressed $(16,21,35)$. This constitutive production of the PHA synthases may be an important mechanism for microorganisms to synthesize PHAs as soon as they sense the imbalance of nutrients.

In addition, we overexpressed either or both of the $p h a E_{\mathrm{Hm}}$ and $p h a C_{\mathrm{Hm}}$ genes in $H$. hispanica ATCC 33960 harboring a highly homologous $p h a E C_{\mathrm{Hh}}$ operon, and we found only coexpression of $p h a E_{\mathrm{Hm}}$ and $p h a C_{\mathrm{Hm}}$ genes resulted in the highest PHB content and PHB yield (Table 3 ) in these transformants. We also introduced $p h a E_{\mathrm{Hm}}$ and/or $p h a C_{\mathrm{Hm}}$ genes into a phaEC-deleted mutant strain, $H$. hispanica PHB-1, and revealed that only coexpression of $p h a E_{\mathrm{Hm}}$ and $p h a C_{\mathrm{Hm}}$ could restore $H$. hispanica $\mathrm{PHB}-1$ with the ability to synthesize $\mathrm{PHB}$ as well as PHA synthase activity. These results clearly confirmed the conclusion that $\mathrm{PhaE}$ and $\mathrm{PhaC}$ should be the active components of the PHA synthase in Haloarcula. However, introduction of $p h a E C_{\mathrm{Hm}}$ genes into the PHA synthase-negative bacterium Cupriavidus necator PHB-4 (32) could not restore its ability to accumulate $\mathrm{PHB}$, although the expression of $\mathrm{PhaE}_{\mathrm{Hm}}$ and $\mathrm{PhaC}_{\mathrm{Hm}}$ was confirmed by Western blotting (data not shown), suggesting that the PHA synthase of $H$. marismortui ATCC 43049 might not be able to fold into its native conformation in the low-salt cytoplasm of the bacterium. It would be interesting to investigate the structure and biochemical features of these novel haloarchaeal PHA synthases in the future.

\section{ACKNOWLEDGMENTS}

We thank W. V. Ng for kindly providing the H. marismortui ATCC 43049 strain and A. Steinbüchel and G. Q. Chen for the C. necator PHB-4 strain.

This work was partially supported by grants from the Ministry of Science \& Technology of China (2004CB719603 and 2006AA09Z401), the National Natural Science Foundation of China (30621005 and 30570029), and the Chinese Academy of Sciences (KSCX2-YW-G-023).

\section{REFERENCES}

1. Baliga, N. S., R. Bonneau, M. T. Facciotti, M. Pan, G. Glusman, E. W. Deutsch, P. Shannon, Y. Chiu, R. S. Weng, R. R. Gan, P. Hung, S. V. Date, E. Marcotte, L. Hood, and W. V. Ng. 2004. Genome sequence of Haloarcula marismortui: a halophilic archaeon from the Dead Sea. Genome Res. 14: 2221-2234.

2. Blaseio, U., and F. Pfeifer. 1990. Transformation of Halobacterium halobium: development of vectors and investigation of gas vesicle synthesis. Proc. Natl. Acad. Sci. USA 87:6772-6776.

3. Bolhuis, H., P. Palm, A. Wende, M. Falb, M. Rampp, F. Rodriguez-Valera, F. Pfeiffer, and D. Oesterhelt. 2006. The genome of the square archaeon Haloquadratum walsbyi: life at the limits of water activity. BMC Genomics 7:169.

4. Cline, S. W., W. L. Lam, R. L. Charlebois, L. C. Schalkwyk, and W. F. Doolittle. 1989. Transformation methods for halophilic archaebacteria. Can. J. Microbiol. 35:148-152.

5. Ellman, G. L. 1959. Tissue sulfhydryl groups. Arch. Biochem. Biophys. 82: $70-77$.

6. Fernandez-Castillo, R., F. Rodriguez-Valera, J. Gonzalez-Ramos, and F. Ruiz-Berraquero. 1986. Accumulation of poly( $\beta$-hydroxybutyrate) by halobacteria. Appl. Environ. Microbiol. 51:214-216.

7. Gerngross, T. U., K. D. Snell, O. P. Peoples, A. J. Sinskey, E. Csuhai, S. Masamune, and J. Stubbe. 1994. Overexpression and purification of the soluble polyhydroxyalkanoate synthase from Alcaligenes eutrophus: evidence for a required posttranslational modification for catalytic activity. Biochemistry 33:9311-9320.

8. Hai, T., S. Hein, and A. Steinbüchel. 2001. Multiple evidence for widespread and general occurrence of type-III PHA synthases in cyanobacteria and molecular characterization of the PHA synthases from two thermophilic cyanobacteria: Chlorogloeopsis fritschii PCC 6912 and Synechococcus sp. strain MA19. Microbiology 147:3047-3060.

9. Hai, T., D. Lange, R. Rabus, and A. Steinbüchel. 2004. Polyhydroxyalkanoate (PHA) accumulation in sulfate-reducing bacteria and identification of a class III PHA synthase (PhaEC) in Desulfococcus multivorans. Appl. Environ. Microbiol. 70:4440-4448.

10. Hezayen, F. F., B. H. Rehm, R. Eberhardt, and A. Steinbüchel. 2000. Polymer production by two newly isolated extremely halophilic archaea: application of a novel corrosion-resistant bioreactor. Appl. Microbiol. Biotechnol 54:319-325.

11. Hezayen, F. F., A. Steinbüchel, and B. H. Rehm. 2002. Biochemical and enzymological properties of the polyhydroxybutyrate synthase from the extremely halophilic archaeon strain 56. Arch. Biochem. Biophys. 403:284-291.

12. Jia, Y., T. J. Kappock, T. Frick, A. J. Sinskey, and J. Stubbe. 2000. Lipases provide a new mechanistic model for polyhydroxybutyrate (PHB) synthases: characterization of the functional residues in Chromatium vinosum $\mathrm{PHB}$ synthase. Biochemistry 39:3927-3936.

13. Jossek, R., R. Reichelt, and A. Steinbüchel. 1998. In vitro biosynthesis of poly(3-hydroxybutyric acid) by using purified poly(hydroxyalkanoic acid) synthase of Chromatium vinosum. Appl. Microbiol. Biotechnol. 49:258-266.

14. Kim, T. K., Y. M. Jung, M. T. Vo, S. Shioya, and Y. H. Lee. 2006. Metabolic engineering and characterization of phaC1 and phaC2 genes from Pseudo- 
monas putida KCTC1639 for overproduction of medium-chain-length polyhydroxyalkanoate. Biotechnol. Prog. 22:1541-1546.

15. Kirk, R. G., and M. Ginzburg. 1972. Ultrastructure of two species of halobacterium. J. Ultrastruct. Res. 41:80-94.

16. Kojima, T., T. Nishiyama, A. Maehara, S. Ueda, H. Nakano, and T. Yamane. 2004. Expression profiles of polyhydroxyalkanoate synthesis-related genes in Paracoccus denitrificans. J. Biosci. Bioeng. 97:45-53.

17. Koller, M., P. Hesse, R. Bona, C. Kutschera, A. Atlić, and G. Braunegg. 2007 Potential of various archae- and eubacterial strains as industrial polyhydroxyalkanoate producers from whey. Macromol. Biosci. 7:218-226.

18. Lam, W. L., and W. F. Doolittle. 1989. Shuttle vectors for the archaebacterium Halobacterium volcanii. Proc. Natl. Acad. Sci. USA 86:5478-5482.

19. Legault, B. A. A Lopez-Lopez, J. C. Alba-Casado, W. F. Doolittle, H Bolhuis, F. Rodriguez-Valera, and R. T. Papke. 2006. Environmental genomics of "Haloquadratum walsbyi" in a saltern crystallizer indicates a large pool of accessory genes in an otherwise coherent species. BMC Genomics $7: 171$.

20. Liebergesell, M., S. Rahalkar, and A. Steinbüchel. 2000. Analysis of the Thiocapsa pfennigii polyhydroxyalkanoate synthase: subcloning, molecular characterization and generation of hybrid synthases with the corresponding Chromatium vinosum enzyme. Appl. Microbiol. Biotechnol. 54:186-194.

21. Liebergesell, M., K. Sonomoto, M. Madkour, F. Mayer, and A. Steinbüchel. 1994. Purification and characterization of the poly(hydroxyalkanoic acid) synthase from Chromatium vinosum and localization of the enzyme at the surface of poly(hydroxyalkanoic acid) granules. Eur. J. Biochem. 226:71-80

22. Liebergesell, M., and A. Steinbüchel. 1992. Cloning and nucleotide sequences of genes relevant for biosynthesis of poly(3-hydroxybutyric acid) in Chromatium vinosum strain D. Eur. J. Biochem. 209:135-150.

23. Lillo, J. G., and F. Rodriguez-Valera. 1990. Effects of culture conditions on poly( $\beta$-hydroxybutyric acid) production by Haloferax mediterranei. Appl. Environ. Microbiol. 56:2517-2521.

24. Liu, S. J., and A. Steinbüchel. 2000. Exploitation of butyrate kinase and phosphotransbutyrylase from Clostridium acetobutylicum for the in vitro biosynthesis of poly(hydroxyalkanoic acid). Appl. Microbiol. Biotechnol. 53: 545-552.

25. Madigan, M. T., and A. Oren. 1999. Thermophilic and halophilic extremophiles. Curr. Opin. Microbiol. 2:265-269.

26. Madison, L. L., and G. W. Huisman. 1999. Metabolic engineering of poly(3hydroxyalkanoates): from DNA to plastic. Microbiol. Mol. Biol. Rev, 63:21-53.

27. Müh, U., A. J. Sinskey, D. P. Kirby, W. S. Lane, and J. Stubbe. 1999. PHA synthase from Chromatium vinosum: cysteine 149 is involved in covalent catalysis. Biochemistry 38:826-837.

28. Pötter, M., M. H. Madkour, F. Mayer, and A. Steinbüchel. 2002. Regulation of phasin expression and polyhydroxyalkanoate (PHA) granule formation in Ralstonia eutropha H16. Microbiology 148:2413-2426.

29. Rehm, B. H. 2003. Polyester synthases: natural catalysts for plastics. Biochem. J. 376:15-33.

30. Sambrook, J., E. F. Fritsch, and T. Maniatis. 1989. Molecular cloning: a laboratory manual, 2nd ed. Cold Spring Harbor Laboratory Press, Cold Spring Harbor, NY.

31. Sartorius-Neef, S., and F. Pfeifer. 2004. In vivo studies on putative ShineDalgarno sequences of the halophilic archaeon Halobacterium salinarum. Mol. Microbiol. 51:579-588.

32. Schlegel, H. G., R. Lafferty, and I. Krauss. 1970. The isolation of mutants not accumulating poly-beta-hydroxybutyric acid. Arch. Mikrobiol. 71:283-294.

33. Schubert, P., N. Krüger, and A. Steinbüchel. 1991. Molecular analysis of the Alcaligenes eutrophus poly(3-hydroxybutyrate) biosynthetic operon: identification of the $\mathrm{N}$ terminus of poly(3-hydroxybutyrate) synthase and identification of the promoter. J. Bacteriol. 173:168-175.

34. Sim, S. J., K. D. Snell, S. A. Hogan, J. Stubbe, C. Rha, and A. J. Sinskey. 1997. PHA synthase activity controls the molecular weight and polydispersity of polyhydroxybutyrate in vivo. Nat. Biotechnol. 15:63-67.

35. Steinbüchel, A., and H. G. Schlegel. 1991. Physiology and molecular genetics of poly(beta-hydroxy-alkanoic acid) synthesis in Alcaligenes eutrophus. Mol. Microbiol. 5:535-542.

36. Stubbe, J., and J. Tian. 2003. Polyhydroxyalkanoate (PHA) hemeostasis: the role of PHA synthase. Nat. Prod. Rep. 20:445-457.

37. Sun, C., Y. Li, S. Mei, Q. Lu, L. Zhou, and H. Xiang. 2005. A single gene directs both production and immunity of halocin C8 in a haloarchaeal strain AS7092. Mol. Microbiol. 57:537-549.

38. Tian, J., A. J. Sinskey, and J. Stubbe. 2005. Detection of intermediates from the polymerization reaction catalyzed by a D302A mutant of class III polyhydroxyalkanoate (PHA) synthase. Biochemistry 44:1495-1503.

39. Tu, D., G. Blaha, P. B. Moore, and T. A. Steitz. 2005. Gene replacement in Haloarcula marismortui: construction of a strain with two of its three chromosomal rRNA operons deleted. Extremophiles 9:427-435.

40. Wendoloski, D., C. Ferrer, and M. L. Dyall-Smith. 2001. A new simvastatin (mevinolin)-resistance marker from Haloarcula hispanica and a new Haloferax volcanii strain cured of plasmid pHV2. Microbiology 147:959-964.

41. Yuan, W., Y. Jia, J. Tian, K. D. Snell, U. Müh, A. J. Sinskey, R. H. Lambalot, C. T. Walsh, and J. Stubbe. 2001. Class I and III polyhydroxyalkanoate synthases from Ralstonia eutropha and Allochromatium vinosum: characterization and substrate specificity studies. Arch. Biochem. Biophys. 394:87-98.

42. Zhou, M., H. Xiang, C. Sun, and H. Tan. 2004. Construction of a novel shuttle vector based on an RCR-plasmid from a haloalkaliphilic archaeon and transformation into other haloarchaea. Biotechnol. Lett. 26:1107-1113. 